\title{
Diversidade de formigas na Floresta Nacional de Chapecó, Santa Catarina, Brasil
}

\author{
Ants diversity in Floresta Nacional de Chapecó in Santa Catarina State, Brazil
}

Junir Antonio Lutinski ${ }^{{ }^{*}}$ Flavio Roberto Mello Garcia ${ }^{I I}$ Cladis Juliana Lutinski ${ }^{\mathrm{I}}$ Samanta Iop

\section{RESUMO}

A mirmecofauna da Floresta Nacional de Chapecó, Gleba I, foi estudada por coletas semanais realizadas de dezembro de 2003 a dezembro de 2004. Foram utilizadas armadilhas do tipo malaise, pit-fall, iscas com sardinha, iscas com glicose, rede de varredura, guarda-chuva entomológico e funil de Berlese. Três constituições vegetais foram amostradas, sendo pinus, eucalipto e Floresta Ombrófila Mista e Floresta Estacional Decidual. Foram capturadas 137.019 espécimes de nove subfamílias, 18 tribos, 36 gêneros e 121 espécies. Os índices de diversidade de Margalef obtidos foram 9,9; 9,7 e 12,6; de Shannon e Wiener 3,0; 3,2 e 3,4 e de equitabilidade de 0,69; 0,73 e 0,74, para as áreas com pinus, eucalipto e mata nativa, respectivamente. Estes resultados indicam uma distribuição mais uniforme na comunidade da mata nativa, caracterizando-se como um importante reservatório espécies de formigas no Oeste catarinense.

Palavras-chave: formicidae, biodiversidade, amostragens.

\section{ABSTRACT}

Mirmecofauna of the Floresta Nacional de Chapecó, Field I, was studied by weekly collections from December of 2003 to December of 2004. Malaise, pit-fall, sardine baits, glucose baits, sweeping net, entomological umbrella and Berlese funnel had been used. Three vegetal constitutions where showed, like pinus, eucalyptus Ombrófila Mista and Estacional Decidual native forests. 137.019 specimens of nine subfamilies, 18 tribes, 36 generas and 121 species were captured. The indices of diversity of Margalef were 9.9; 9.7 and 12.6; of Shannon and Wiener 3.0; 3.2 and 3.4 and of equitability of $0.69 ; 0.73$ and 0.74 , for the areas with pinus, eucalyptus and native forest, respectively. These results indicate a more uniform distribution in the community of the native forest, characterizing itself as an important reservoir species of ants in the West region of the Santa Catarina State, Brazil.

Key words: formicidae, biodiversity, samplings.

\section{INTRODUÇÃO}

A Floresta Nacional de Chapecó é a segunda maior Floresta do IBAMA de Santa Catarina, sendo a única a possuir Floresta Estacional Decidual no Estado. Esta reserva constitui-se em um dos últimos remanescentes dessa floresta preservada no Oeste catarinense, sendo importante realizar pesquisas para conhecer a diversidade de espécies deste ecossistema.

As formigas constituem um dos grupos de insetos mais conhecidos e estudados (HÖLLDOBLER \& WILSON, 1990), existindo cerca de 12.030 espécies. No entanto, estimativas mostram a possibilidade de existir até 574 gêneros e 21.847 espécies (AGOSTI \& JOHNSON, 2003).

A distribuição das formigas na região Tropical Americana não obedece padrão uniforme para gêneros e espécies. Existem espécies endêmicas restritas a pequenas regiões e outras encontradas da América do Norte à América do Sul. Além disso, a distribuição e o endemismo das espécies podem ser alterados à medida que se intensificam os trabalhos de inventariamento (LATTKE, 2003).

O conhecimento taxonômico de formicídeos na região Oeste de Santa Catarina restringe-se, até o momento, aos resultados de um trabalho de mais de seis décadas de coletas nãosistematizadas, realizadas por Fritz Plaumann, que resultou no registro de 179 espécies de formigas, em 57 gêneros (SILVA, 1998).

\footnotetext{
'Laboratório de Entomologia, Universidade Comunitária Regional de Chapecó (UnoChapecó), Centro de Ciências Agroambientais e de Alimentos, CP 747, 89809-000, Chapecó, SC, Brasil. E-mail: lutinski@ibest.com.br. *Autor para correspondência.

IISetor de pesquisa, Universidade La Salle, Canoas, RS, Brasil.
} 
Este trabalho teve por objetivo conhecer e avaliar a diversidade e a estrutura das diferentes comunidades da mirmecofauna da Floresta Nacional de Chapecó.

\section{MATERIAL E MÉTODOS}

O estudo foi conduzido na Gleba I da Floresta Nacional de Chapecó (FLONA), localizada no município de Guatambú, SC, na localidade da Fazenda Zandavalli (27 05’ 50”S; 5246’ 40’W), em três áreas, com cerca de 5 ha cada uma. A área um, constituída por uma plantação de pinus (Pinus taeda Linnaeus e Pinus elliottii Engelm), com idade aproximada de 35 anos, apresenta sub-bosque de vegetação nativa bem diversificada, caracterizando estágio avançado de sucessão ecológica. Na área dois predomina uma plantação de eucalipto (Eucalyptus saligna Smith e Eucalyptus grandis Hill), com idade de aproximadamente cinco anos, tendo sub-bosque esparso e composto exclusivamente por gramíneas, o que indica perturbação ambiental que tende a agravar-se à medida que as plantas crescem e limitam a entrada de luz. Na área três a cobertura vegetal é de Floresta Ombrófila Mista e Floresta Estacional Decidual, não existindo relatos de desmatamento.

As amostragens foram semanais no período de dezembro de 2003 a dezembro de 2004. Nas coletas foram utilizadas armadilhas do tipo ativo, caso da rede de varredura, guarda-chuva entomológico e funil de BerleseTullgren e as armadilhas passivas pit-fall, malaise, iscas com sardinha e iscas com glicose invertida(BESTELMEYER et al., 2000; SARMIENTO, 2003).

Uma pré-identificação das formigas foi realizada no laboratório de Entomologia da UNOCHAPECÓ, a partir das chaves taxonômicas propostas por FERNÁNDEZ (2003). A confirmação dos táxons foi realizada pela comparação com espécimes da coleção do Laboratório com base em BOLTON(2003).

A diversidade de formicídeos de cada uma das áreas foi mensurada por meio dos índices de diversidade de Margalef e de Shannon e Wiener (PINTO-COELHO, 2000).

O grau de aproximação das comunidades (similaridade) foi analisado por meio de um gráfico deCluster, com base nas freqüências absolutas dos registros feitos para cada espécie, em cada comunidade, e construído a partir do programa STATISTICA 6.1.

Todos os cálculos dos índices de diversidade, de equitabilidade e de similaridade, para as áreas inventariadas, foram realizados a partir do número de registros de cada espécie, para cada área e método, uma vez que as características sociais das formigas podem afetar estas análises, quando realizadas sobre os números absolutos de espécimes coletados.

\section{RESULTADOS E DISCUSSÃO}

Comunidade de formigas da FLONA

Durante os estudos foram capturadas 137.019 espécimes de formigas pertencentes a nove subfamílias, 18 tribos, 36 gêneros e 121 espécies.

Na área de mata nativa, foram capturadas 102 espécies que representam 20 a mais do que na área com eucalipto e 22 espécies a mais do que na área com pinus. Em nível de Gênero, Tribo e Subfamília, ocorreu uma alta similaridade entre as três áreas (Tabelas 1,2 e 3).

Os resultados obtidos refletem a importância da mata nativa como um reservatório da fauna de formigas e de outros invertebrados. As 121 espécies de formigas capturadas representam 67,59\% das 179 espécies relatadas com ocorrência na região(SILVA, 1999).

Como a fauna de formigas pode estar correlacionada com a de outros invertebrados (SILVESTRE et al., 2003), a preservação desta FLONA tem importância para manter e dispersar espécies para outras áreas.

Nas três áreas de estudo, ocorreram simultaneamente 61 espécies de formigas. Aárea com pinus contou com 10 espécies de formigas de ocorrência exclusiva, que foram Myrmelachista sp. 1, Basiceros convexiceps Mayr, 1887, Octostruma rugifera Mayr, 1887, Crematogaster limata Fr. Smith, 1858, Acanthognathus sp., Strumigenys cultriger Mayr, 1887, Hypoponera sp. 6, Hypoponera sp. 7, Pachycondyla crenata (Roger, 1861)e Pachycondyla sp. 1. Os limitados recursos disponibilizados pela vegetação de pinus indica que estas espécies de formigas conseguem ocupar nichos criados pela vegetação de sub-bosque presente na área.

Octostruma, Basiceros e Strumigenys são formigas predadoras especializadas e vivem associadas à serrapilheira (SILVESTRE et al., 2003). A presença destas na área com pinus permite inferir que uma população diversificada de invertebrados faz parte desta comunidade. Já a presença de formigas como Labidus, nas três áreas estudadas, demonstra a tolerância destas espécies às perturbações ambientais como ocorre na área com eucalipto. Labidus apresenta ampla distribuição na região Oeste de Santa Cartarina, como pode se verificado nos trabalhos de SILVA\& SILVESTRE (2000) e LUTINSKI \& GARCIA(2005).

Sete espécies ocorreram somente na área com eucalipto, sendo que: Acanthosthichus serratulus Fr. Smith, 1858, Heteroponera microps Borgmeier, 1957, Crematogaster crinosa Mayr, 1862, Pheidole sp. 9, Dinoponera australis Emery, 1901, Pseudomyrmex sp. 2e Pseudomyrmex sp. 3. Crematogaster, Dinoponera e 
Tabela 1 - Registros das espécies de formigas em áreas com pinus, eucaliptos e mata nativa na Floresta Nacional de Chapecó. Dezembro de 2003 a dezembro de 2004.

\begin{tabular}{|c|c|c|c|}
\hline Táxon & Pinus & Eucalipto & Mata nativa \\
\hline \multicolumn{4}{|l|}{ 1) Subfamília Cerapachyinae - Tribo Acanthostichini } \\
\hline Acanthosthichus serratulus Fr. Smith, 1858 & - & 1 & - \\
\hline \multicolumn{4}{|l|}{ 2) Subfamília Dolichoderinae - Tribo Dolichoderini } \\
\hline Dorymyrmex brunneus Forel, 1908 & 39 & 651 & 48 \\
\hline Dorymyrmex sp. & 6 & 53 & 8 \\
\hline Linepithema humile Mayr, 1868 & 85 & 27 & 78 \\
\hline Linepithema sp. 1 & 6 & 5 & 4 \\
\hline Linepithema sp. 2 & 1 & 1 & 10 \\
\hline Linepithema sp. 3 & 40 & 15 & 24 \\
\hline Linepithema sp. 4 & _- & _ & 1 \\
\hline Tapinoma atriceps Emery, 1888 & 2 & 42 & _ \\
\hline Tapinoma melanocephalum Fabricius, 1793 & - & - & 1 \\
\hline \multicolumn{4}{|l|}{ 3) Subfamília Ecitoninae - Tribo Ecitonini } \\
\hline Eciton burchellii (Westwood, 1842) & 2 & _ & 1 \\
\hline Labidus coecus (Latreille, 1802) & 7 & 69 & 31 \\
\hline Labidus praedator (Fr. Smith, 1858) & 18 & 38 & 45 \\
\hline Nomamyrmex hartigii (Westwood, 1842) & - & 2 & 1 \\
\hline \multicolumn{4}{|l|}{ 4) Subfamília Ectatomminae - Tribo Ectatommini } \\
\hline Ectatomma edentatum Roger, 1863 & 3 & 29 & 27 \\
\hline Gnamptogenys striatula Mayr, 1884 & 9 & 1 & 71 \\
\hline \multicolumn{4}{|l|}{ 5) Subfamília Formicinae - Tribo Camponotini } \\
\hline Camponotus crassus Mayr, 1862 & 70 & 342 & 69 \\
\hline Camponotus diversipalpus Santschi, 1922 & 30 & 82 & 69 \\
\hline Camponotus mus Roger, 1863 & _- & _ & 1 \\
\hline Camponotus rufipes (Fabricius, 1775) & 253 & 329 & 73 \\
\hline Camponotus sericeiventris G.-Méneville, 1838 & 2 & - & 7 \\
\hline Camponotus sp. 1 & 6 & 26 & 5 \\
\hline Camponotus sp. 2 & 6 & 6 & 51 \\
\hline Camponotus sp. 3 & 54 & 46 & 35 \\
\hline Camponotus sp. 4 & 6 & 6 & 11 \\
\hline Camponotus sp. 5 & 15 & 14 & 6 \\
\hline Camponotus sp. 6 & 4 & 1 & 2 \\
\hline Camponotus sp. 7 & 8 & 48 & 22 \\
\hline Camponotus sp. 8 & 1 & 1 & 3 \\
\hline Camponotus sp. 9 & 2 & 1 & 1 \\
\hline Camponotus sp. 10 & 3 & 5 & 1 \\
\hline Camponotus sp. 11 & - & _- & 1 \\
\hline Camponotus sp. 12 & - & 1 & 1 \\
\hline Camponotus sp. 13 & - & 4 & 2 \\
\hline Camponotus sp. 14 & - & _- & 1 \\
\hline Camponotus sp. 15 & - & - & 1 \\
\hline \multicolumn{4}{|l|}{ Tribo Plagiolepidini } \\
\hline Brachymyrmex sp. & 2 & 1 & 1 \\
\hline Myrmelachista sp. 1 & 2 & - & - \\
\hline
\end{tabular}

Pheidole são formigas freqüentemente relatadas fazem parte de diferentes constituições vegetais no Oeste catarinense, conforme pode ser observado nos estudos de SILVA\&SILVESTRE(2000),eLUTINSKI\&GARCIA(2005).

Um total de 22 espécies incidiram somente na área de mata nativa, perfazendo a maior fauna exclusiva dntre as três áreas estudadas, sendo: Linepithema sp. 4,
Tapinoma melanocephalum Fabricius, 1793, Camponotus mus Roger, 1863, Camponotus sp. 11, Camponotus sp. 14, Camponotus sp. 15, Myrmelachista sp. 4, Paratrechina sp. 2, Acanthoponera mucronata (Roger, 1860), Cephalotes sp. 3, Crematogaster sp. 8, Pheidole sp. 2, Pheidole sp. 14, Solenopsis sp. 6, Acromyrmex disciger (Mayr, 1887), Acromyrmex sp., Belanopelta curvata Mayr,

Ciência Rural, v.38, n.7, out, 2008. 
Tabela 2 - Registros das espécies de formigas em áreas com pinus, eucaliptos e mata nativa na Floresta Nacional de Chapecó. Dezembro de 2003 a dezembro de 2004.

\begin{tabular}{|c|c|c|c|}
\hline Táxon & Pinus & Eucalipto & Mata nativa \\
\hline Myrmelachista sp. 2 & 2 & 1 & - \\
\hline Myrmelachista sp. 3 & 208 & 73 & 263 \\
\hline Myrmelachista sp. 4 & - & - & 1 \\
\hline Paratrechina fulva (Mayr, 1862) & 3 & 1 & 2 \\
\hline Paratrechina longicornis Latreille, 1802 & 2 & 1 & 2 \\
\hline Paratrechina sp. 1 & 1 & 1 & 8 \\
\hline Paratrechina sp. 2 & 0 & - & 1 \\
\hline \multicolumn{4}{|l|}{ 6) Subfamília Heteroponerinae - Tribo Heteroponerini } \\
\hline Acanthoponera mucronata (Roger, 1860) & - & - & 1 \\
\hline Heteroponera microps Borgmeier, 1957 & - & 1 & - \\
\hline \multicolumn{4}{|l|}{ 7) Subfamília Myrmicinae - Tribo Basicerotini } \\
\hline Basiceros convexiceps Mayr, 1887 & 1 & - & - \\
\hline Octostruma rugifera Mayr, 1887 & 1 & - & - \\
\hline \multicolumn{4}{|l|}{ Tribo Blepharidattini } \\
\hline Wasmannia auropunctata Roger, 1863 & 7 & 1 & 2 \\
\hline Wasmannia sp. & - & 1 & 2 \\
\hline Tribo Cephalotini - Cephalotes pusillus (Klug, 1824) & 1 & - & 1 \\
\hline Cephalotes sp. 2 & - & 2 & 2 \\
\hline Cephalotes sp. 3 & - & - & 4 \\
\hline Procryptocerus sp. & - & 1 & 2 \\
\hline \multicolumn{4}{|l|}{ Tribo Crematogastrini } \\
\hline Crematogaster acuta (Fabricius, 1804) & 2 & 6 & 1 \\
\hline Crematogaster corticicola Mayr, 1887 & 6 & 10 & 40 \\
\hline Crematogaster crinosa Mayr, 1862 & - & 6 & - \\
\hline Crematogaster limata Fr. Smith, 1858 & 1 & - & - \\
\hline Crematogaster nigropilosa Mayr, 1870 & 15 & 219 & 17 \\
\hline Crematogaster sp. 6 & 3 & 5 & 16 \\
\hline Crematogaster sp. 7 & 1 & 30 & 3 \\
\hline Crematogaster sp. 8 & - & - & 1 \\
\hline Crematogaster sp. 9 & - & $\overline{2}$ & 1 \\
\hline \multicolumn{4}{|l|}{ Tribo Dacetini } \\
\hline Acanthognathus ocellatus Mayr, 1887 & 0 & 1 & 1 \\
\hline Acanthognathus sp & 1 & - & - \\
\hline Strumigenys cultriger Mayr, 1887 & 2 & - & - \\
\hline Strumigenys sp. & _ & 2 & 1 \\
\hline \multicolumn{4}{|l|}{ Tribo Myrmicini - } \\
\hline Pogonomyrmex naegelli (Fabricius 1805) & 5 & 305 & 6 \\
\hline Pogonomyrmex sp. & 1 & 2 & 1 \\
\hline Tribo Pheidolini - Pheidole sp. 1 & 244 & 201 & 126 \\
\hline Pheidole sp. 2 & - & - & 6 \\
\hline Pheidole sp. 3 & 38 & 38 & 66 \\
\hline Pheidole sp. 4 & 442 & 366 & 315 \\
\hline Pheidole sp. 5 & 187 & 121 & 193 \\
\hline Pheidole sp. 6 & 10 & 10 & 11 \\
\hline Pheidole sp. 8 & 7 & 7 & 6 \\
\hline Pheidole sp. 9 & - & 2 & - \\
\hline
\end{tabular}

1887, Hypoponera foeda (Forel, 1912), Hypoponera sp. 4, Hypoponera sp. 5, Pachycondyla sp. 2 e Pseudomyrmex sp. 4. Este fato pode ser explicado pelas condições de preservação das características originais da vegetação, pelos recursos disponíveis e pelas relações entre a fauna e a flora.

Sete espécies de formigas ocorreram simultaneamente nas áreas com pinus e com mata nativa, sendo: Eciton burchellii (Westwood, 1842), Camponotus sericeiventris Guérin-Méneville, 1838, Cephalotes pusillus

Ciência Rural, v.38, n.7, out, 2008. 
Tabela 3 - Registros das espécies de formigas em áreas com pinus, eucaliptos e mata nativa na Floresta Nacional de Chapecó. Dezembro de 2003 a dezembro de 2004.

\begin{tabular}{|c|c|c|c|}
\hline Táxon & Pinus & Eucalipto & Mata nativa \\
\hline Pheidole sp. 10 & 464 & 142 & 292 \\
\hline Pheidole sp. 11 & 28 & 31 & 39 \\
\hline Pheidole sp. 13 & _ & 1 & 1 \\
\hline Pheidole sp. 14 & _- & _ & 1 \\
\hline Pheidole sp. 15 & 7 & 2 & 13 \\
\hline \multicolumn{4}{|l|}{ Tribo Solenopsidini } \\
\hline Solenopsis saevissima (Fr. Smith, 1855) & 14 & 188 & 32 \\
\hline Solenopsis sp. 2 & 35 & 9 & 15 \\
\hline Solenopsis sp. 3 & 19 & 165 & 69 \\
\hline Solenopsis sp. 4 & 1 & 18 & 11 \\
\hline Solenopsis sp. 5 & - & 2 & 4 \\
\hline Solenopsis sp. 6 & _ & _ & 1 \\
\hline Solenopsis sp. 7 & 5 & - & 4 \\
\hline Tribo Attini - Acromyrmex disciger (Mayr, 1887) & - & - & 3 \\
\hline Acromyrmex niger (Fr. Smith, 1858) & 6 & 53 & 16 \\
\hline Acromyrmex subterraneus Forel, 1893 & 1 & 1 & 3 \\
\hline Acromyrmex sp. & - & _- & 1 \\
\hline Apterostigma pilosum Mayr, 1865 & 2 & 62 & 8 \\
\hline Apterostigma sp. 2 & 1 & - & 1 \\
\hline Apterostigma sp. 3 & 1 & 4 & 1 \\
\hline Atta sexdens Linnaeus, 1758 & 29 & 107 & 241 \\
\hline Mycocepurus goeldii Forel, 1893 & 12 & 92 & 48 \\
\hline \multicolumn{4}{|l|}{ 8) Subfamília Ponerinae - Tribo Ponerini } \\
\hline Belanopelta curvata Mayr, 1887 & - & _- & 1 \\
\hline Dinoponera australis Emery, 1901 & - & $\overline{4}$ & _- \\
\hline Hypoponera distinguenda Emery 1890 & 48 & 6 & $\overline{15}$ \\
\hline Hypoponera foeda (Forel, 1912) & - & - & 5 \\
\hline Hypoponera opacior (Forel, 1893) & 9 & 6 & 19 \\
\hline Hypoponera sp. 4 & - & - & 6 \\
\hline Hypoponera sp. 5 & - & - & 2 \\
\hline Hypoponera sp. 6 & 2 & - & _- \\
\hline Hypoponera sp. 7 & 2 & - & - \\
\hline Odontomachus chelifer (Latreille, 1802) & 44 & 2 & $\overline{2}$ \\
\hline Pachycondyla crenata (Roger, 1861) & 1 & _- & _- \\
\hline Pachycondyla harpax (Fabricius, 1804) & 13 & - & 14 \\
\hline Pachycondyla striata Fr. Smith, 1858 & 131 & 26 & 219 \\
\hline Pachycondyla villosa (Fabricius, 1804) & 16 & _- & 7 \\
\hline Pachycondyla sp. 1 & 1 & - & _- \\
\hline Pachycondyla sp. 2 & - & - & 1 \\
\hline \multicolumn{4}{|c|}{ 9) Subfamília Pseudomyrmecinae - Tribo Pseudomyrmecini } \\
\hline Pseudomyrmex flavidulus (Fr. Smith, 1858) & 0 & 2 & 1 \\
\hline Pseudomyrmex gracilis (Fabricius, 1804) & 80 & 62 & 57 \\
\hline Pseudomyrmex sp. 1 & 7 & 24 & 1 \\
\hline Pseudomyrmex sp. 2 & - & 1 & - \\
\hline Pseudomyrmex sp. 3 & - & 1 & - \\
\hline Pseudomyrmex sp. 4 & - & _- & 1 \\
\hline
\end{tabular}

(Klug, 1824), Solenopsis sp. 7, Apterostigma sp. 2, Pachycondyla harpax (Fabricius, 1804) e Pachycondyla villosa (Fabricius, 1804). Estas coberturas vegetais, que estão em estágio final de sucessão, onde a serrapilheira e o sub-bosque estão bem formados, embora as constituições vegetais destas duas áreas difiram em diversidade e disponibilidade de alimento e locais de nidificação, têm características bióticas que favorecem a presença de formigas.

Ciência Rural, v.38, n.7, out, 2008. 
Estrutura e diversidade das comunidades de formigas

Os índices de diversidade de Margalef foram 9,9; 9,7 e 12,6, enquanto que os índices da diversidade de Shannon e Wiener foram 3,0; 3,2 e 3,4, para as áreas com pinus, eucalipto e mata nativa, respectivamente (Figura 1).

O maior valor da equitabilidade foi encontrado para a área com mata nativa, indicando uma distribuição mais uniforme da fauna de formicídeos nesta comunidade. Os valores encontrados foram 0,74; 0,73 e 0,69, respectivamente para as áreas com mata nativa, eucalipto e pinus. Segundo PINTO-COELHO (2000), o índice de equitabilidade é considerado indicativo de uniformidade na distribuição das espécies no local avaliado.

Houve proximidade espacial entre as comunidades de formigas das áreas com pinus e eucalipto, embora sejam culturas em estágios diferentes de sucessão. A condição de equilíbrio de mata nativa, sem dúvida, é o fator que contribui positivamente para o estabelecimento de uma comunidade de formigas mais diversificada.

Fatores como temperatura, umidade, precipitação e disponibilidade de alimentos podem atuar diretamentena diversidade de formigas (FERREIRA, 1986). Quanto maior a complexidade da vegetação, maior a diversidade da comunidade de formigas que pode ser sustentada (ANDERSEN, 1984; SOARES et al., 2003). Embora estudos de correlação entre estes fatores não tenham sido objeto do estudo, a disponibilidade de alimento e os locais para nidificação, que são mais abundantes e diversificados na área de mata nativa, explicam a maior diversidade de espécies encontrada neste nicho.
Similaridade entre as três comunidades avaliadas

Os valores da diversidade indicaram um distanciamento da comunidade de formigas da área com mata nativa em relação às demais, embora uma análise da similaridade aproximou as comunidades de formigas das áreas com pinus e mata nativa. Assim, a análise de Cluster coloca em condições de igualdade estrutural as duas comunidades, distanciando-as da comunidade de formigas da área com eucalipto (Figura 2).

A similaridade entre as populações da área com floresta nativa e com pinus pode ser explicada pela condição de povoamento da plantação com pinus, embora a monocultura ecologicamente representa uma barreira para o estabelecimento da diversidade da flora e da fauna. $\mathrm{O}$ povoamento propiciou o estabelecimento de um subbosque e uma serrapilheira capaz de exercer um papel sobre a fauna de formicídeos semelhante àquela exercida pela mata nativa.

Embora a equitabilidade não tenha demonstrado uma diferença estrutural significativa para a distribuição dos registros realizados para as espécies de cada comunidade, estes foram mais numerosos na área com eucalipto. De acordo com LARA (1992), esta situação pode ser explicada pelo fato de que, em áreas em melhores condições de preservação, as relações interespecíficas são mais numerosas, resultando em populações mais equilibradas e, em geral, com maior número de espécimes, quando comparadas com comunidades em estado de maior grau de alteração. Populações maiores, de espécies mais generalistas, podem ter contribuído para o maior número

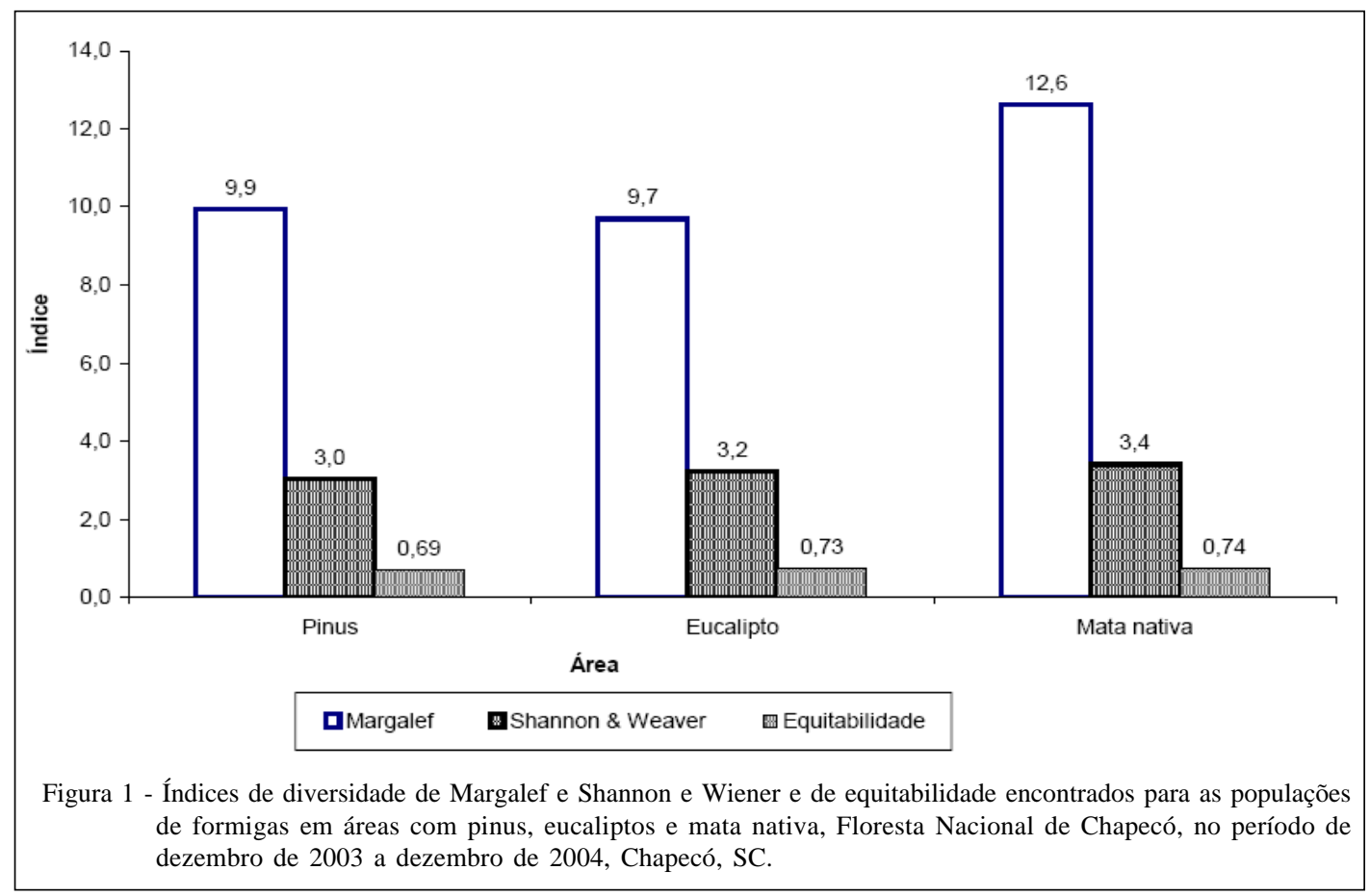

Ciência Rural, v.38, n.7, out, 2008. 


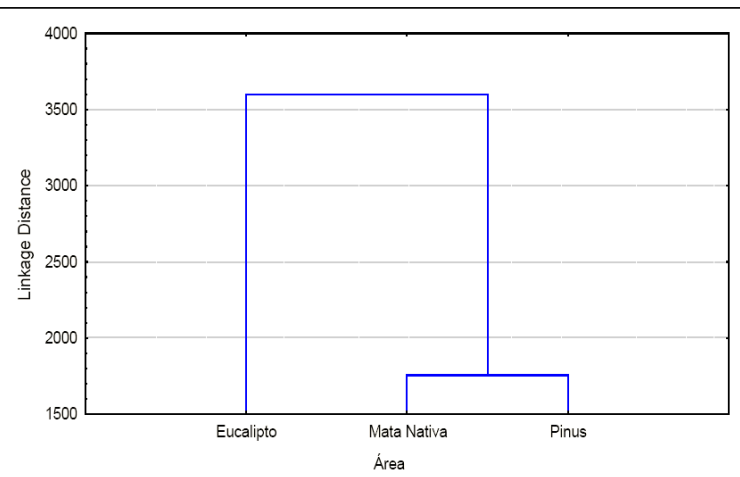

Figura 2 - Análise de similaridade entre as populações de formigas em áreas de pinus, eucalipto e mata nativa na Floresta Nacional de Chapecó, no período de dezembro de 2003 a dezembro de 2004, Chapecó, SC.

de espécimes na comunidade de formigas na área com eucalipto.

A similaridade observada entre as comunidades das áreas com pinus e mata nativa possibilita inferir que, embora na mata nativa ocorra $25 \%$ mais espécies que na área com pinus, estas duas comunidades compartilham maior número de fatores bióticos em relação a área com eucalipto.

\section{CONCLUSÕES}

A Floresta Nacional de Chapecó caracteriza-se como um importante reservatório de espécies de Formicidae.

Os índices de diversidade e equitabilidade evidenciam maior equilíbrio entre as espécies na área de mata nativa, enquanto que a similaridade das populações de formigas nas áreas de mata nativa e pinus sugere que as espécies compartilham fatores bióticos.

\section{AGRADECIMENTO}

À administração da FLONA, pela autorização da pesquisa, que possibilitou o estudo, e à UNOCHAPECÓ, pelo auxilio técnico e financeiro.

Ao Professor Dr. Benedito Cortês Lopes do Departamento de Ecologia e Zoologia da UFSC, pelo auxílio na determinação de espécies.

\section{REFERÊNCIAS}

AGOSTI, D.; JOHNSON, N.F. La nueva taxonomía de hormigas. In: FERNANDEZ, F. Introducción a las hormigas de la región neotropical. Bogotá, Colombia: Instituto de investigación de recursos biológicos Alexander von Humboldt, 2003. p.45-48.

ANDERSEN, A.N. Community organization of ants in the Victorian Mallee. Victorian Naturalist, Melbourne, v.101, p.248-251, 1984. BESTELMEYER, B.T. et al. Field techniques for the study of ground-dwelling ants. Ants: standard of methods for measuring and monitoring biodiversity. Washington: Smithsonian Institution, 2000. p.122-144.

BOLTON, B. Synopsis and classification of Formicidae. Gainesville, Florida: The American Entomological Institute, 2003. 370p.

FERNÁNDEZ, F. Introducción a las hormigas de la región neotropical. Bogotá, Colombia: Instituto de investigación de recursos biológicos Alexander von Humboldt, 2003. 418p.

FERREIRA, M.F.B. Análise faunística de Formicidae (Insecta: Hymenoptera) em ecossistemas naturais e agroecossistemas na região de Botucatu, SP. 1986. $73 \mathrm{f}$. Dissertação (Mestrado) - Universidade Estadual Paulista "Júlio de Mesquita Filho”, Brasil.

HÖLLDOBLER, B.; WILSON, E.O. The ants. Cambridge: Belknap, Harvard University, 1990. 732p.

LARA, F.M. Princípios de entomologia. São Paulo, Brasil: Ícone, 1992. 331p.

LATTKE, J.E. Biogeografía de las hormigas neotropicales. In: FERNANDEZ, F. Introducción a las hormigas de la región neotropical. Bogotá, Colombia: Instituto de investigación de recursos biológicos Alexander von Humboldt, 2003. p.65-85.

LUTINSKI, J.A.; GARCIA, F.R.M. Análise faunística de Formicidae (Hymenoptera: Apocrita) em ecossistema degradado no município de Chapecó, Santa Catarina. Biotemas, Florianópolis, v.18, n.2, p.73-86, 2005.

PINTO-COELHO, R.M. Fundamentos em ecologia. Porto Alegre: Artmed, 2000. 252p.

SARMIENTO, C.E. Metodologías de captura y estudio de las hormigas. In: FERNANDEZ, F. Introducción a las hormigas de la región neotropical. Bogotá, Colombia: Instituto de investigación de recursos biológicos Alexander von Humboldt, 2003. p.201-210.

SILVA, R.R. A coleção entomológica do Museu Fritz Plaumann. Biotemas, Florianópolis, v.11, n.2, p.157-164, 1998.

SILVA, R.R. Formigas (Hymenoptera: Formicidae) do Oeste de Santa Catarina: histórico das coletas e lista atualizada das espécies do Estado de Santa Catarina. Biotemas, Florianópolis, v.12, n.2, p.75-100, 1999.

SILVA, R.R.; SILVESTRE, R. Diversidade de formigas (Hymenoptera: Formicidae) em Seara, oeste de Santa Catarina. Biotemas, Florianópolis, v.13, n.2, p.85-105, 2000.

SILVESTRE, R. et al. Grupos funcionales de hormigas: el caso de los gremios del cerrado. In: FERNANDEZ, F. Introducción a las hormigas de la región neotropical. Bogotá, Colombia: Instituto de investigación de recursos biológicos Alexander von Humboldt, 2003. p.113-148.

SOARES, I.M.F. et al. Comunidades de formigas (Hymenoptera: Formicidae) em uma "ilha” de floresta Ombrófila Serrana em região da Caatinga (Ba, Brasil). Acta Biológica Leopoldencia, São Leopoldo, v.25, n.2, p.197-204, 2003.

Ciência Rural, v.38, n.7, out, 2008. 